\title{
Advances in Managing Long Term Ecological Research Data
}

\author{
William K. Michener ${ }^{a^{* *}}$ \\ a College of University Libraries and Learning Science, University of New Mexico, \\ Albuquerque, NM 87131, USA
}

Long Term Ecological Research (LTER) studies have proliferated over the past several decades and new programs such as the U.S. National Ecological Observatory Network (NEON) ensure that such studies will continue for decades to come (Michener and Waide 2009, Michener 2015). Concepts and practices underpinning LTER ecoinformatics were reviewed previously (Michener et al. 2011, Michener and Jones 2012). Given the importance of LTER research to our ecological understanding and the pace of informatics advances over the past several years, it is important to periodically take stock of recent and relevant LTER ecoinformatics achievements. This special issue focuses on seven exemplary ecoinformatics programs that have significantly advanced the state-of-the-art of LTER ecoinformatics.

First, the information management program at the North Temperate Lakes (NTL) LTER site in Wisconsin USA is described by Gries and colleagues (Gries et al. 201_; this issue). The NTL project has created one of the world's most scientifically comprehensive and important collections of data on lakes and their surroundings. The NTL information management program is notable for being an early adopter of both community standards and emerging technologies, as well as for developing robust custom data entry and management applications that facilitate fish, zooplankton and water chemistry research. In addition to highlighting recent advances, the paper also summarizes the evolution of the NTL information management program and provides a valuable summary of some of the lessons learned in the process.

Second, the Provenance Aware Synthesis Tracking Architecture data repository is described by Servilla and colleagues (Servilla et al. 201_; this issue). The U.S. LTER network has accumulated more than 5,000 valuable data sets related to ecological patterns and processes in a broad range of ecosystems since its inception approximately 35 years ago. Yet, the value of these data cannot be realized unless the data are of the highest quality and are easily discoverable and interpretable. The PASTA-based LTER Network Information System provides many valuable functions for LTER researchers. In particular, data are readily discoverable and available through a single web-interface and, importantly, products include provenance metadata for tracking source and derived data. Digital Object Identifiers are assigned to those data products that are deposited in the repository after meeting structural and semantic quality requirements. In addition to describing the enhanced features of the repository, the paper summarizes the characteristics of the repository's holdings and discusses re-use of the long-term data.

\footnotetext{
*E-mail address: wmichene@unm.edu
} 
Third, a unified data management system for distributed experiments is described by Lind (Lind 201_; this issue). Ecologists recognize that ecological processes such as decomposition and primary production differ markedly in their nature and pace across the diverse ecosystems and habitats of the world. Developing better understanding of general trends and their variances requires that researchers perform distributed experiments across the diversity of ecosystems. This paper describes the database system developed to support the Nutrient Network (NutNet) that currently holds up to 10 years of data from more than 90 sites worldwide. The high scientific productivity associated with NutNet is in large part due to the innovative relational database schema and associated processes that support efficient storage, reconciliation and storage of alternative taxonomies, and that facilitate easy recombination and synthesis of data.The paper includes the relational database schema and documents how the approach could be applied to and benefit other distributed ecological experiments.

Fourth, a cloud-based platform that supports reproducible science is described and demonstrated by Guru and colleagues (Guru et al. 201_; this issue). Technologies that support more transparent and reproducible science are important not only for increasing trust in the results, but also for enabling the original researchers and others to both reproduce results and to grow knowledge by easily adding in new data and evolving new and more sophisticated analyses based on existing algorithms. This paper describes a cloud-based Collaborative Environment for Ecosystem Science Research and Analysis (CoESRA) infrastructure that allows one to build, execute and share complex analytical workflows. The paper further demonstrates the creation, sharing and re-use of a Kepler scientific workflow that reproduces an IUCN Red List of Ecosystems Assessmentundertaken on Mountain Ash forests in Australia.

Fifth, the Ecological Metadata Language (EML) Congruency Checker (ECC) system is described by O'Brien and colleagues (O'Brien et al. 201_; this issue). High quality and comprehensive metadata are essential for interpretability and re-use of long-term data. Quality assessments of metadata have heretofore been principally a time-consuming manual activity. This paper describes the ECC system that is used by the U.S. LTER Network Information System to automatically screen data packages for completeness and quality, and to verify that the data typing and formats documented in the metadata match those of the data entities. The authors describe overall data improvements since adoption of the ECC system and identify future plans for continuing evolution of the tool.

Sixth, a comprehensive data management plan for acquiring ocean carbonate chemistry data associated with $\mathrm{pH}$ sensors is described by Rivest and colleagues (Rivest et al. 201_; this issue). Increasingly, complex sensors and sensor arrays capture environmental data. This paper presents best practices for sensor deployment, calibration, data processing and quality control. The paper presents a workflow pipeline for using a specific $\mathrm{pH}$ sensor technology and associated data management procedures and, importantly, provides a useful template for designing data management for other complex sensors.

Seventh, a prototype system for multilingual data discovery of international LTER data is described by Vanderbilt and colleagues (Vanderbilt et al. 201_; this 
issue). As LTER networks are implemented globally researchers are challenged in discovering relevant data that are described in different languages. This paper presents a prototype web-services-based system for searching international LTER data repositories that is based on multilingual thesauri. The authors demonstrate that the approach in combination with online automatic translation tools enables scientists to assess the suitability of discovered data for particular uses.

The subject matter covered in the articles included in this special issue mesh well with the evolving nature of LTER research. Specifically, the continued growth of LTER data within existing research networks and the global growth of LTER networks, the addition of new community collaborations like NutNet as well as new environmental observatories like NEON amplify the need for new approaches to discovering, managing and ensuring quality of the associated large and diverse data holdings. Five years from now, we can expect to see significant advances in supporting transparency and reproducibility of data and results, and much greater attention paid to automated quality assurance and quality control, especially in relation to the ensuing deluge of data from environmental sensors in the field.

\section{Acknowledgments}

Support for the author and editor was provided by the U.S. National Science Foundation (ACI- 1430508; IIA-1301346).

\section{References}

Gries, C., Gahler, M.R., Hanson, P.C., Kratz, T.R., Stanley, E.H., 201_. Information management at the North Temperate Lakes Long-Term Ecological Research site successful support of research in a large, diverse, and long running project. Ecol. Inform.

Guru, S., Hanigan, I.C., Nguyen, H.A., Burns, E., Stein, J., Blanchard, W., Lindenmayer, D., Clancy, T., 201_. Development of a cloud-based platform for reproducible science: a case study of an IUCN Red List of Ecosystems Assessment. Ecol. Inform.

Lind, E.M., 201_. Unified data management for distributed experiments: a model for collaborative grassroots scientific networks. Ecol. Inform.

Michener, W.K., 2015. Ecological data sharing. Ecol. Inform. 29:33-44, doi:10.1016/j.ecoinf.2015.06.010

Michener, W.K., Jones, M.B., 2012. Ecoinformatics: supporting ecology as a dataintensive science. Trends Ecol. Evol. 27:85-93.

Michener, W.K., Waide, R.B., 2009. The evolution of collaboration in ecology: lessons from the United States Long Term Ecological Research Program. Pages 297-310 in Olson, G.M., Zimmerman, A., Bos, N. (Eds.), Scientific Collaboration on the Internet, MIT Press, Boston. 
Michener, W.K., Porter, J., Servilla, M., Vanderbilt, K., 2011. Long term ecological research and information management. Ecol. Inform. 6:13-24.

O'Brien, M., Costa, C., Servilla, M., 201_. Ensuring the quality of data packages in the LTER network data management system. Ecol. Inform.

Rivest, E., O’Brien, M., Kapsenberg, L., Gotschalk, C.C., Blanchette, C.A., Hoshijima, U., Hofmann, G.E., 201_. Beyond the benchtop and the benthos: dataset management planning and design for time series of ocean carbonate chemistry associated with Durafet@-based pH sensors. Ecol. Inform.

Servilla, M., Brunt, J., Costa, D., McGann, J., Waide, R., 201_. The contribution and reuse of LTER data in the Provenance Aware Synthesis Tracking Architecture (PASTA) data repository. Ecol. Inform.

Vanderbilt, K., Porter, J.H., Lu, S-S., Bertrand, N., Blankman, D., Guo, X., He, H., Henshaw, D., Jeong, K., Kim, E-S., Lin, C-C., O’Brien, M., Osawa, T., Tuama, E.O., Su, W., Yang, H. 201_.A prototype system for multilingual data discovery of international long-term ecological research (ILTER) network data. Ecol. Inform. ______. 